\title{
Lugol's solution-induced painless thyroiditis
}

\author{
Ji Wei Yang and Jacques How \\ Division of Endocrinology and Metabolism, Department of Medicine, McGill University Health Centre, \\ Montreal, Quebec, Canada
}

Correspondence should be addressed to J How

Email

jacques.how@mcgill.ca

\section{Summary}

Lugol's solution is usually employed for a limited period for thyroidectomy preparation in patients with Graves' disease and for the control of severe thyrotoxicosis and thyroid storm. We describe a rare case of Lugol's solution-induced painless thyroiditis. In November 2014, a 59-year-old woman was prescribed Lugol's solution four drops per day for the alleviation of menopausal symptoms. She was referred to our clinic in June 2015 for fatigue, hair loss, and a 20-lb weight loss without thyroid pain or discomfort. Physical examination revealed a normal thyroid gland. On 7 May 2015, laboratory tests revealed a suppressed thyroid-stimulating hormone (TSH) $0.01 \mathrm{U} / \mathrm{L}$ with elevated free T4 $3.31 \mathrm{ng} / \mathrm{dL}$ (42.54 pmol/L). Repeat testing on 25 May 2015 showed spontaneous normalization of the free thyroid hormone levels with persistently low TSH $0.10 \mathrm{U} / \mathrm{L}$. Following these results, a family physician prescribed methimazole $10 \mathrm{mg}$ PO TID and very soon after, the TSH concentration rose to $>100 \mathrm{U} / \mathrm{L}$ along with subnormal free T4 and T3 levels. Methimazole was promptly discontinued, namely within 18 days of its initiation. Over the course of the next few months, the patient spontaneously achieved clinical and biochemical euthyroidism. To our knowledge, this is a unique case of painless thyroiditis induced by Lugol's solution, which has not been reported before. Lugol's solution is a short-term medication given for the preparation of thyroidectomy in patients with Graves' disease and for the control of severe thyrotoxicosis. lodine excess can cause both hyperthyroidism and hypothyroidism. Rarely, Lugol's solution can cause acute painless thyroiditis.

\section{Learning points:}

- Lugol's solution is used for thyroidectomy preparation in patients with Graves' disease and for the control of severe thyrotoxicosis and thyroid storm.

- Iodine excess can cause both hypothyroidism and thyrotoxicosis. Thyroid glands with an underlying pathology are particularly susceptible to the adverse effect of iodine.

- The prolonged off-label use of Lugol's solution can be harmful. Rarely, Lugol's solution can cause acute painful thyroiditis.

\section{Background}

Lugol's solution is commonly used for the preparation of thyroidectomy in patients with Graves' disease as well as for the control of severe thyrotoxicosis and thyroid storm. Prolonged use of Lugol's solution in current clinical practice is most unusual. Here, we report a case of Lugol's solution-induced painless thyroiditis.

\section{Case presentation}

A 59-year-old patient was referred to our thyroid clinic in June 2015. Her personal and family histories were unremarkable for thyroid disease. Her regular medications were atorvastatin, estradiol, progesterone, salbutamol and a combination of formoterol and mometasone. In November 2014, a physician prescribed Lugol's solution four drops 
per day for the relief of menopausal symptoms. More recently, the patient complained of fatigue, hair loss, and a 20-lbs weight loss despite normal appetite. She denied thyroid pain or discomfort. Physical examination revealed a tired-looking patient, without signs of thyroid-associated ophthalmopathy. Blood pressure was $130 / 78 \mathrm{mmHg}$, with a normal heart rate of 70 beats per minute. Thyroid examination revealed a normal and non-tender gland without vascular bruits. The patient's palms were cool to touch.

\section{Investigation}

On 7 May 2015, laboratory tests revealed a suppressed thyroid-stimulating hormone (TSH) $0.01 \mathrm{U} / \mathrm{L}$ with elevated free T4 level $3.31 \mathrm{ng} / \mathrm{dL}$ (42.54 pmol/L). An earlier TSH on 5 December 2014 was normal at $1.55 \mathrm{U} / \mathrm{L}$. Interestingly, repeated testing on 25 May 2015 showed spontaneous normalization of free T4 levels to $0.95 \mathrm{ng} / \mathrm{dL}(12.20 \mathrm{pmol} / \mathrm{L})$, along with normal free T3 levels of $2.89 \mathrm{pg} / \mathrm{mL}$ (4.45 pmol/L); TSH remained low at 0.10 U/L. Anti-TPO and anti-thyroglobulin antibodies were positive at $102.30 \mathrm{IU} /$ $\mathrm{mL}$ and $5.7 \mathrm{kIU} / \mathrm{L}$ respectively, while anti-TSH receptor antibody titers were negative. Despite these findings, the patient's primary physician prescribed methimazole $10 \mathrm{mg}$ PO TID on 4 June 2015. A thyroid ultrasound performed on 8 June 2015 showed a normal-sized, mildly hypoechoic, heterogeneous thyroid gland, with images suggestive of on-going thyroiditis. Laboratory tests done at our centre on 23 June 2015 showed markedly elevated TSH >100 U/L with reduced levels of free T4 $0.26 \mathrm{ng} / \mathrm{dL}(3.40 \mathrm{pmol} / \mathrm{L})$ and free T3 $1.54 \mathrm{pg} / \mathrm{mL}(2.37 \mathrm{pmol} / \mathrm{L})$.

\section{Treatment}

No therapeutic intervention was required for this patient since her thyroid dysfunction resolved spontaneously. Methimazole was discontinued on 23 June, upon finding the laboratory results consistent with severe hypothyroidism.

\section{Outcome and follow-up}

Follow-up laboratory tests on 13 July 2015 showed that the TSH had dropped to $47.28 \mathrm{U} / \mathrm{L}$; and free $\mathrm{T} 4$ and $\mathrm{T} 3$ levels were $0.41 \mathrm{ng} / \mathrm{dL}(5.30 \mathrm{pmol} / \mathrm{L})$ and $2.07 \mathrm{pg} / \mathrm{mL}$ $(3.19 \mathrm{pmol} / \mathrm{L})$ respectively. Serial thyroid function tests confirmed continuous spontaneous improvement and by early December 2015, the patient achieved biochemical euthyroidism. Anti-TPO and anti-thyroglobulin antibody titers normalized concomitantly with the recovery of euthyroidism.

\section{Discussion}

This patient's earliest thyroid presentation at primary care level was thought to be hyperthyroidism due to Graves' disease, and indeed, the family physician started the patient on methimazole right away. The evolution of the thyroid data and the absence of TSH receptor antibodies however did not support Graves' disease. Instead, spontaneous normalization of the thyroid function tests prompted the possibility of iodine-induced thyrotoxicosis. In this context, the history of Lugol's solution treatment for six months was informative and provocative. Thyroid uptake scan was not done since it was no longer relevant by the time that she was referred to us.

Iodine excess can cause both hypothyroidism and hyperthyroidism. Wolff and Chaikoff first described the transient decrease in thyroid hormone synthesis in response to iodine load in rats in the 1940s. With continuous administration of iodide, an escape phenomenon, manifested by the resumption of normal thyroid hormone synthesis, is observed (1). This adaptive response is mediated by a decrease in the sodium iodide symporter, which causes decreased active transport of iodine into the thyroid gland (2). Failure to escape the Wolff-Chaikoff effect can result in iodine-induced hypothyroidism in susceptible individuals (3). At the other end of the spectrum, iodineinduced thyrotoxicosis, known as the Jod-Basedow phenomenon, can also occur, especially in individuals with goitres in iodine-deficient regions $(4,5)$.

Lugol's iodine, a solution containing $100 \mathrm{mg} / \mathrm{mL}$ of potassium iodide and $50 \mathrm{mg} / \mathrm{mL}$ of iodine, was developed in 1829 by the French physician Jean Guillaume Auguste Lugol (1786-1851). François Magendie (1783-1855), a French physiologist, considered by many as a pioneer of experimental physiology, popularized the use of iodine in medicine. In those early days, iodine was used for a number of pathologies, including scrofula, more commonly known as tuberculosis cervical lymphadenitis (6). In current clinical practice, Lugol's solution is given preoperatively to minimize intraoperative blood loss during thyroidectomy in patients with Graves' disease, and for the treatment of severe thyrotoxicosis and thyroid storm. In both instances, Lugol's solution is given for a short period. We are not aware of the use of Lugol's solution in the control of menopausal symptoms, and our search of the literature did not uncover any publications substantiating this practice. 
Our interpretation for our patient's clinical picture is that of a painless thyroiditis, induced by the uncommon prolonged use of Lugol's solution. To our knowledge, This is a most unusual case, which has not been previously reported. In 1955, Edmunds reported four cases of painful thyroiditis after ingestion of a potassium iodide solution (7). In all cases, thyroid pain began within $48 \mathrm{~h}$ of administration and resolved within $72 \mathrm{~h}$ of discontinuation of the medication. Thyroid function test results were not reported. The author concluded that the painful thyroid afflictions likely represented an allergic reaction to the potassium iodide. Calvi and Daniels described a case of destructive thyroiditis in a euthyroid 30-year-old patient after cardiac catheterization, which was believed to be secondary to the direct toxicity of the iodinated contrast material (8). The patient received a total iodine load of $177.6 \mathrm{~g}$ over $24 \mathrm{~h}$, with acute onset of fever, neck pain and leukocytosis within the next $48 \mathrm{~h}$. Laboratory tests confirmed thyrotoxicosis. Finally, Leustean et al. observed a case of Jod-Basedow effect, which exacerbated a pre-existing Graves' thyrotoxicosis, after inadvertent prolonged use of Lugol's solution for 30 days (9). The presence of the initially positive tests for antiTPO and anti-thyroglobulin antibodies in our patient was at first interpreted as reflective of a concomitant chronic lymphocytic thyroiditis. It is well known that a gland afflicted with an underlying autoimmune thyroiditis is more susceptible to the adverse effects of excess exogenous iodine. However, spontaneous normalization of the antibody titers over time in parallel with recovery of normal thyroid function is more suggestive of a secondary immune response to the destructive thyroiditis, brought on by the prolonged iodine load from Lugol's solution.

Interestingly, our patient informed us that her brother and her sister-in-law, who live in another distant province of Canada, were independently also prescribed Lugol's solution by their family physician for prolonged period for the treatment of a non-thyroidal disorder. We therefore suspect that there exists a more widespread offlabel use of Lugol's solution, and our case illustrates the potential harm of such exposure.

Lugol's iodine is commonly used for brief periods (days) in the setting of pre-operative thyroidectomy preparation as well as severe thyrotoxicosis and thyroid storm. It is rare to see it employed for long periods of time as it was the case in our patient. Inadvertent prolonged use can induce thyrotoxicosis and exacerbate pre-existing thyrotoxicosis, especially in patients whose primary indication for Lugol's solution is the control of a severe thyrotoxicosis. Rarely, Lugol's solution can cause acute painful thyroiditis. Our case illustrates the potential harm of prescribing Lugol's solution for an off-label indication.

\section{Declaration of interest}

The authors report no conflicts of interest. This work was in part presented at the 2015 Canadian Society of Endocrinology and Metabolism/Canadian Diabetes Association Professional Conference \& Annual Meetings, held in Vancouver, British Columbia, Canada, 30 November-3 December 2015.

\section{Funding}

This research did not receive any specific grant from any funding agency in the public, commercial or not-for-profit sector.

\section{Patient consent}

Written signed consent has been obtained from the patient for publication of the submitted article.

Author contribution statement

Both authors contributed equally to the manuscript.

\section{References}

1 Wolff J \& Chaikoff IL 1948 Plasma inorganic iodide as a homeostatic regulator of thyroid function. Journal of Biological Chemistry 174 555-564.

2 Eng PH, Cardona GR, Fang SL, Previti M, Alex S, Carrasco N, Chin WW \& Braverman LE 1999 Escape from the acute WolffChaikoff effect is associated with a decrease in thyroid sodium/iodide symporter messenger ribonucleic acid and protein. Endocrinology 140 3404-3410. (doi:10.1210/endo.140.8.6893)

3 Leung AM \& Braverman LE 2012 Iodine-induced thyroid dysfunction. Current Opinion in Endocrinology: Diabetes and Obesity 19 414-419. (doi:10.1097/MED.0b013e3283565bb2)

4 Martino E, Safran M, Aghini-Lombardi F, Rajatanavin R, Lenziardi M, Fay M, Pacchiarotti A, Aronin N, Macchia E, Haffajee C, et al. 1984 Environmental iodine intake and thyroid dysfunction during chronic amiodarone therapy. Annals of Internal Medicine 10 28-34. (doi:10.7326/0003-4819-101-1-28)

5 Bogazzi F, Bartalena L \& Martino E 2010 Approach to the patient with amiodarone-induced thyrotoxicosis. Journal of Clinical Endocrinology and Metabolism 95 2529-2535. (doi:10.1210/jc.2010-0180)

6 Kelly FC 1961 Iodine in medicine and pharmacy since its discovery-1911-1961. Proceedings of the Royal Society of Medicine 54 831-836.

7 Edmunds HT 1955 Acute thyroiditis from potassium iodide. BMJ 1 354. (doi:10.1136/bmj.1.4909.354-a)

8 Calvi L \& Daniels GH 2011 Acute thyrotoxicosis secondary to destructive thyroiditis associated with cardiac catheterization contrast dye. Thyroid 21 443-449. (doi:10.1089/thy.2010.0300)

9 Leustean L, Preda C, Ungureanu MC, Danila R, Mogos V, Stefanescu C $\&$ Vulpoi C 2014 Jod-Basedow effect due to prolonged use of lugol solution-case report. Revista Medico-Chirurgicala a Societatii De Medici Si Naturalisti Din Iasi 118 1013-1017.

Received in final form 23 February 2017

Accepted 3 May 2017 